\title{
What the Physical Activity Community Can Do for Climate Action and Planetary Health
}

\author{
Rodrigo Reis, Ruth F. Hunter, Leandro Garcia, and Deborah Salvo
}

\begin{abstract}
We are experiencing a planetary tipping point with global warming, environmental degradation, and losses in biodiversity. The burdens of these changes fall disproportionately on poor and marginalized populations. Physical activity promotion strategies need to be aligned with climate action commitments, incorporating the Intergovernmental Panel on Climate Change scenarios in physical activity action plans. The promotion strategies must consider equity a core value and promote physical activity to the most vulnerable populations so that they are protected from the ill-health impacts of a changing climate.
\end{abstract}

Keywords: climate change, climate adaptation, public health, systems thinking, health promotion

The philosopher Francis Bacon said that "in order for the light to shine so brightly, the darkness must be present." The release of the Sixth Assessment Report of the Intergovernmental Panel on Climate Change ${ }^{1}$ and the 2021 report of the Lancet Countdown on health and climate change ${ }^{2}$ has made unequivocal that humankind's darkest days are present and the chances for a brighter future for us are rapidly decreasing. The overwhelming evidence indicates that nearly all regions of the world are experiencing dramatic changes in weather and extreme climate events. Though human actions still have the potential to determine our future, the need to adapt the human existence to an already changed climate is also urgent.

The call for a global action to promote at-scale physical activity strategies has made the case for communities to be physically active as a means of contributing to a more sustainable planet. ${ }^{3}$ Recently, Salvo et $\mathrm{al}^{4}$ demonstrated in simulated scenarios that climate mitigation may benefit from multiple scale-up physical activity promotion strategies, largely due to shifts toward more active forms of travel and recreation. The global physical activity community has a major role to play in this regard. Unfortunately, adapting to a changing climate has rarely been considered in the context of physical activity promotion. Preparing physical activity infrastructures and policies for a warming world must be considered a priority as physical activity inequities in some of the hottest and poorest countries will only deepen. ${ }^{2}$

The need for preparing health policies and strategies for human-environment interactions has been obvious during the COVID-19 pandemic. Globally, communities have struggled to deliver their health programs and address the urgency and scale of the COVID-19 pandemic. Unfortunately, the lack of preparedness, capacity, and the struggle to quickly adapt to a new reality has caused a major burden among the poorest- - those already suffering from chronic diseases and the older adult populations. That was also the case for physical activity, which when promoted and encouraged through adequate spaces and programs for active travel and recreation, particularly among those most in need, have helped

Reis and Salvo are with Prevention Research Center, Brown School, Washington University in St Louis, St Louis, MO, USA. Reis is also with the Urban Management, Pontifical Catholic University of Parana, Curitiba, Brazil. Hunter is with the Centre for Public Health, Queen's University Belfast, Institute of Clinical Sciences B, Royal Victoria Hospital, Belfast, Northern Ireland, United Kingdom. Garcia is with the Centre for Public Health, Queen's University Belfast, Belfast, United Kingdom. Reis (reis.rodrigo@wustl.edu) is corresponding author. to alleviate health and societal burdens of the pandemic. Hence, it is paramount that physical activity promotion strategies are ready to rapidly adapt to similarly disruptive events in the future.

We argue that the health of the population and our collective behavior influence the health of the planet, and at the same time, the health of the planet influences our health-the relationship is bidirectional and symbiotic in nature. In essence, the health and well-being of the human population and of the planet cannot be disassociated. The physical activity community must better understand how climate change influences physical activity and subsequently population health. In tandem, it is imperative that any solutions to this (ie, climate change) and many other public health challenges faced by the global physical activity community "cause no harm" to our planet at the very least, and preferably, must substantially contribute toward climate change mitigation. Thus, we also must better understand the interdependencies between climate change, physical activity, population, and planetary health.

Viewing physical activity promotion through a systemsoriented lens, using systems-thinking principles and approaches and complexity science methods, will help us identify solutions that promote physical activity and a range of health and societal benefits, avoid unintended consequences and harms to the health of people and the planet more widely, and adapt and respond to uncertain and changing future contexts. The physical activity community needs to study, develop, and implement sustainable, orchestrated actions that push systemic shifts toward the future we all want. These solutions should also be guided by the principles and practices of social innovations allowing communities to generate ideas and solutions collaboratively to adapt at-scale physical activity promotion strategies for a changing climate.

Finally, it is paramount that physical activity promotion strategies are aligned with climate action commitments, such as being carbon neutral, incorporating climate resiliency into all changes in physical activity infrastructure, incorporating the Intergovernmental Panel on Climate Change scenarios in physical activity action plans, considering equity a core value, and promoting physical activity to the most vulnerable populations so they are protected from the ill-health impacts of a changing climate.

The physical activity community has much to offer to climate action and planetary health. Thus, we must engage more actively in the discussions and actions that are indispensable to ensure brighter days for all. 


\section{Acknowledgments}

This work was supported by the Centers for Disease Control and Prevention (number U48DP006395) and the Foundation for Barnes-Jewish Hospital. The findings and conclusions in this paper are those of the authors and do not necessarily represent the official positions of the Centers for Disease Control and Prevention. The authors declare not having any conflict of interest.

\section{References}

1. Masson-Delmotte VP, Zhai A, Pirani SL, et al, eds. IPCC, 2021: Climate Change 2021: The Physical Science Basis. Contribution of
Working Group I to the Sixth Assessment Report of the Intergovernmental Panel on Climate Change. Cambridge University Press.

2. Romanello M, McGushin A, Di Napoli C, et al. The 2021 report of the Lancet Countdown on health and climate change: code red for a healthy future [published online ahead of print October 20, 2021]. Lancet. doi:10.1016/S0140-6736(21)01787-6

3. World Health Organization. Global Action Plan on Physical Activity 2018-2030: More Active People for a Healthier World. Geneva, Switzerland: World Health Organization; 2018.

4. Salvo D, Garcia L, Reis RS, et al. Physical activity promotion and the united nations sustainable development goals: building synergies to maximize impact [published online ahead of print July 13, 2021]. J Phys Act Health. doi:10.1123/jpah.2021-0413 